\title{
Concepto indígena de inteligencia en etnias de México
}

\author{
Joaquín Figueroa Cuevas ${ }^{1}$ y Laura Hernández Guzmán² \\ Universidad Nacional Autónoma de México
}

Se realizaron tres estudios dedicados a explorar las nociones de inteligencia en etnias mexicanas. Los resultados del primer estudio indicaron que los profesores mestizos y nahuas definen la inteligencia infantil en términos tecnológicos, propios de países industrializados. Sin embargo, cuando los profesores nahuas la definieron desde la perspectiva étnica incluyeron proporciones equivalentes de conceptos sociales y tecnológicos. En un segundo estudio se indagó el concepto que poseían las madres nahuas, quienes subrayaron la importancia de los aspectos sociales de la inteligencia. En el tercer estudio, profesores indígenas de diferentes regiones étnicas definieron el concepto de inteligencia congruentemente con los profesores nahuas del primer estudio. De los tres estudios, se concluye que las nociones de inteligencia se relacionan con el grado de contacto cultural de quien la define.

Palabras clave: inteligencia social, etnias, indígenas, niños, educación bilingiie.

\section{Indigenous concept of intelligence in Mexican ethnic groups}

Three studies were conducted to explore the notion of intelligence in Mexican ethnic groups. Results of the first study showed that mestizo and nahua teachers defined child intelligence in technological terms, as in industrialized countries. However, indigenous teachers, defining intelligence from the ethnic viewpoint, stressed its social in the same proportion as its technological aspects. In the second study, the concept of intelligence of nahua mothers emphasized the importance of social aspects of intelligence. In the third study, bilingual teachers from different ethnic regions defined intelligence in equivalent terms as nahua teachers from the first study. Conclusions from the three studies point to a concept of intelligence related to the level of cultural contact of the person defining it.

Keywords: social intelligence, ethnic groups, indigenous, children, bilingual education.

1 Maestro y licenciado en Psicología por la UNAM. Diplomados en Cuestión Indígena y Antropología Jurídica. Profesor de la facultad de psicología UNAM. Autor de más de 25 artículos en las áreas de psicología cultural y educación indígena. Correo electrónico: luxkanan@correo.unam.mx

2 Doctorada en Psicología y Desarrollo Humano por la Universidad de Kansas, EE.UU. Licenciada en Psicología por la UNAM. Profesora/investigadora de la Facultad de Psicología. Autora de más de 50 artículos de investigación en las áreas de psicología educativa y de la salud. Presidenta de la Sociedad Mexicana de 

En vista de la práctica generalizada que simplemente toma pruebas e instrumentos de evaluación de la inteligencia construidos en países industrializados para aplicarlos en sociedades en vías de desarrollo (Serpell, 1984), son diversos los autores que subrayan la importancia de contar con información acerca de la noción de inteligencia que poseen diversas culturas (Sternberg \& Grigorenko, 1997; Sternberg et al., 2002). Para muchas de éstas, existe una gran diversidad étnica que se sustenta principalmente en una población indígena ${ }^{3}$ considerable que, por su propia identificación, lengua, instituciones diferenciadas y símbolos colectivos, cuenta con culturas que, por su núcleo histórico, son distintas a la matriz cultural occidental.

Entre los problemas asociados con la reproducción mecánica de instrumentos de evaluación de la inteligencia en contextos diferentes al de países desarrollados, destaca el empleo de procedimientos y técnicas de evaluación intelectual extraños al ambiente cultural de los sujetos evaluados y, por lo tanto, insensibles para medir las habilidades de algunos niños intelectualmente competentes de países en vías de desarrollo (Cole \& Scribner, 1977; Price-Williams, Gordon \& Ramirez, 1969). El uso de instrumentos insensibles afecta decisiones que, en muchos casos, actúan en detrimento de las personas señaladas equivocadamente.

Psicología, y Editora General del International Journal of Psychology. Correo electrónico: lher@servidor.unam.mx

3 Los criterios para definir a los pueblos indígenas y determinar el tamaño de su población es un problema mundial. En México oficialmente se utiliza como criterio censal a las personas hablantes mayores de 5 años, lo que produce una subestimación de la población indígena. Actualmente se tiende a aceptar la definición establecida en el acuerdo 169 de la OIT como criterio práctico. 
De acuerdo con Sternberg y Grigorenko (1997) y Sternberg et al. (2002), las pruebas de inteligencia traducidas o construidas con base en conceptos anglo europeos evalúan habilidades adquiridas en el sistema educativo, desestimando otras habilidades que posiblemente las culturas de origen valoren como importantes y, por ende, las promuevan en sus niños.

Una estrategia que se ha propuesto, entre otras (PriceWilliams et al., 1969), para evitar este problema, es basar la construcción de pruebas de inteligencia en los criterios y valores de la inteligencia identificados por los pueblos indígenas y/o el uso de habilidades culturalmente relevantes (véase, por ejemplo, Church \& Katigbak, 1988; Grigorenko et al., 2001; Kathuria \& Serpell, 1998).

La literatura de investigación sobre este tema arroja consistentemente una noción de inteligencia, aún en culturas distantes, con una connotación social. Esta inteligencia social contrasta con la inteligencia tecnológica subyacente a la construcción de pruebas en países industrializados, y se ha definido como comportamientos y representaciones en favor del bienestar de las comunidades y la interacción social al interior de éstas. Por otra parte, la definición de inieligencia tecnológica se refiere al pensamiento objetivo y analítico con predominancia de la manipulación de objetos, el control sobre el medio físico y los avances tecnológicos.

La gran mayoría de los estudios no ha encontrado correlación entre las mediciones tradicionales de la inteligencia y las definiciones indígenas de niño inteligente (Dasen et al., 1985; Grigorenko et al., 2001; Serpell, 1977). En general, los conceptos no industrializados de la inteligencia se parecen más a los conceptos de sabiduría que a su representación occidental (Yang \& Sternberg, 1997).

Los datos sugieren, en su mayor parte, que las nociones indígenas de la inteligencia no poseen una correspondencia inequívoca con la perspectiva tecnológica occidental. Según Berry 
(1984), son muchas las culturas que no comparten la visión de la inteligencia como rápida analítica y puramente cognoscitiva.

En las nociones indígenas de la inteligencia predomina un valor primordial a los atributos intelectuales de orden social como el respeto y la conformidad social, la obediencia, la responsabilidad, la cortesía y naturaleza servicial, que se dirigen al beneficio colectivo más que a la ganancia individual. Por ejemplo, para los kipsigis de Kenia, el concepto de inteligencia se refiere a la obediencia y a lo servicial, pero también a la responsabilidad, traducida en la habilidad y capacidad para interpretar con rapidez situaciones complejas de interacción personal (Super, 1983). Mediante tareas de diferencial semántico, empleando escalas de 25 adjetivos bipolares, Wober (1974) encontró que el significado del término obugezi, en el idioma luganda de la etnia africana baganda, incluye aspectos que en inglés se refieren separadamente a inteligencia y a sabiduría, pero con la asociación adicional de conducta respetuosa y de conformidad social.

Otros autores han contrastado las estimaciones de los adultos con la ejecución de los niños en las pruebas de inteligencia convencionales. Los resultados de un estudio en la etnia baloué de Costa de Marfil revelaron tanto contenidos sociales como tecnológicos. El cálculo de la inteligencia de los niños, realizado por miembros de la comunidad, se comparó contra los resultados de la aplicación de una batería de 19 pruebas piagetanas (Dasen et al., 1985).

Estos hallazgos coinciden con los de otro estudio (Serpell, 1977) en Zambia, en el que se encontró la predominancia de los contenidos sociales en el concepto de inteligencia. Identificó este autor como atributos de la inteligencia los significados listo, sabio, cooperativo, obediente, responsable y respetuoso. Los resultados de las pruebas psicométricas tradicionales correlacionaron con el desempeño escolar, pero coincidieron parcialmente con las concepciones indígenas en una investigación de Church y Katigbak 
(1988) con niños campesinos de Filipinas, cuya lengua materna es el tagalog. Éstas, además de incluir el pensamiento agudo, consideraban habilidades cognoscitivas, sociales y motivacionales que suelen desestimarse en las pruebas tradicionales.

A diferencia de los resultados de la mayoría de estudios realizados con población africana o asiática, Klein, Freeman y Millet (1973) hallaron una alta correspondencia entre la concepción guatemalteca de inteligencia y el desempeño de los niños en la resolución de una batería de pruebas psicométricas. Encontraron como equivalente de la palabra inteligencia el término listura. Un niño listo se consideraba independiente, con facilidad verbal, buena memoria, viveza y un alto nivel de actividad física.

Aún para los estadounidenses, la noción de inteligencia incluye también predominante o parcialmente atributos sociales. Por ejemplo, de acuerdo con Sternberg (1985) y Sternberg, Conway, Ketron y Bernstein (1981), ninguna concepción teórica de la inteligencia es capaz de incluir las características que los norteamericanos comunes identifican como atributos intelectuales. De acuerdo con estos autores, la concepción de inteligencia de los norteamericanos comunes distingue tres grandes componentes: habilidades para solucionar problemas prácticos, habilidad verbal y competencia social. En contraste, la concepción teórica de la inteligencia, en la que se basa la construcción de las pruebas tradicionales, incluye como categorías la inteligencia verbal, la habilidad para solucionar problemas y las habilidades prácticas, y deja fuera la competencia social.

Esta línea de investigación ha sugerido, además, diferencias en la noción de inteligencia entre personas de la misma etnia que mantienen diferentes grados de contacto con la cultura occidental en relación con su nivel de escolaridad (Dasen et al., 1985; Wober, 1974). En este mismo sentido, Grigorenko et al. (2001) refieren que, en comparación con los compañeros y adultos de la 
misma comunidad, los profesores valoran más los atributos relacionados con las habilidades escolares.

Aparentemente no existen estudios documentados que investiguen el concepto de inteligencia en etnias mexicanas. El presente trabajo además de explorar la noción de inteligencia en la etnia nahua, se propuso explorar si a mayor educación y contacto con la cultura occidental se daba un concepto más tecnológico de inteligencia. Asimismo, se propuso corroborar si los resultados en la etnia nahua se repetían en otras etnias mexicanas. Por inteligencia social se entendió la base de los comportamientos y representaciones que favorecen el bienestar de las comunidades y facilita las interacciones sociales; por ejemplo, la ayuda, la responsabilidad, la obediencia. Por otra parte, inteligencia tecnológica se definió como la base del pensamiento objetivo y analítico en el que predomina la manipulación de objetos, el control sobre el medio físico y los avances tecnológicos. Se realizaron tres estudios con el propósito de explorar las nociones de inteligencia, en términos de su significado tecnológico y social, de: a) profesores mestizos y nahuas en la etnia nahua, b) personas de la comunidad nahua (madres de familia), y c) profesores pertenecientes a otras etnias mexicanas.

\section{ESTUDIO 1}

Conceptos de inteligencia tecnológica y social en profesores indígenas

\section{Metodología}

\section{Participantes}

Participaron los 27 profesores rurales de la comunidad de Xilocuautla en el municipio de Huachinango, a doscientos 
kilómetros al noroeste de la Ciudad de México. Dieciocho eran mestizos que sólo hablaban español e impartían clases en español, de los cuales 9 a niños mestizos y 9 a niños mestizos y nahuas, y otros 9 profesores nahuas que hablaban más frecuentemente el náhuatl que el español, vivían en la comunidad nahua de Xilocuautla, compartían las costumbres de ésta (alimentación, compadrazgo, boda, cuidado de niños, etc.) e impartían clases en náhuatl a niños nahuas. Este municipio es el que cuenta con el mayor número de indígenas en la sierra norte del estado de Puebla (Chamoux, 1987). Los habitantes de Xilocuautla son en su mayoría nahuas, dedicados al cultivo del maíz, chile, tomate y col.

\section{Procedimiento}

Los 27 profesores definieron lo que es para ellos un niño o niña inteligente (concepto personal). T‘mbién se les pidió, en forma individual y por escrito, que describieran lo que era un niño inteligente para la comunidad nahua (concepto de la comunidad). Cada profesor podía ofrecer cuantos conceptos encontrara para describir niño inteligente.

\section{Confiabilidad entre jueces}

Cuatro jueces independientes e ignorantes de los propósitos del estudio clasificaron la lista de conceptos proporcionados por los profesores en tres categorías: a) inteligencia tecnológica, que alude a características racionales, individuales e independientes de comportamientos afectivos y culturales, caracterizada por los siguientes términos: lógico, comprende, sistemático, razona, analítico; b) inteligencia social, que remite a comportamientos generados en relaciones interpersonales como colaborador, 
respetuoso, responsable, obediente, participativo; y c) otras, categoría que engloba términos como bueno, fuerte, sano, etc.

Se calculó la confiabilidad entre jueces, dividiendo la suma de los acuerdos entre la suma de acuerdos más desacuerdos. Se obtuvo una confiabilidad de .98 .

\section{Resultados}

Se contabilizó la totalidad de los conceptos que ofreció cada profesor, aunque se repitieran algunos de un profesor a otro. En total, los 27 profesores generaron 81 conceptos, 21 proporcionados por los profesores mestizos de acuerdo con el concepto personal y 19 de acuerdo con la noción de la comunidad nahua. Por su parte, los nueve profesores nahuas ofrecieron 20 de acuerdo con su concepto personal y 21 de acuerdo con la noción de la comunidad nahua.

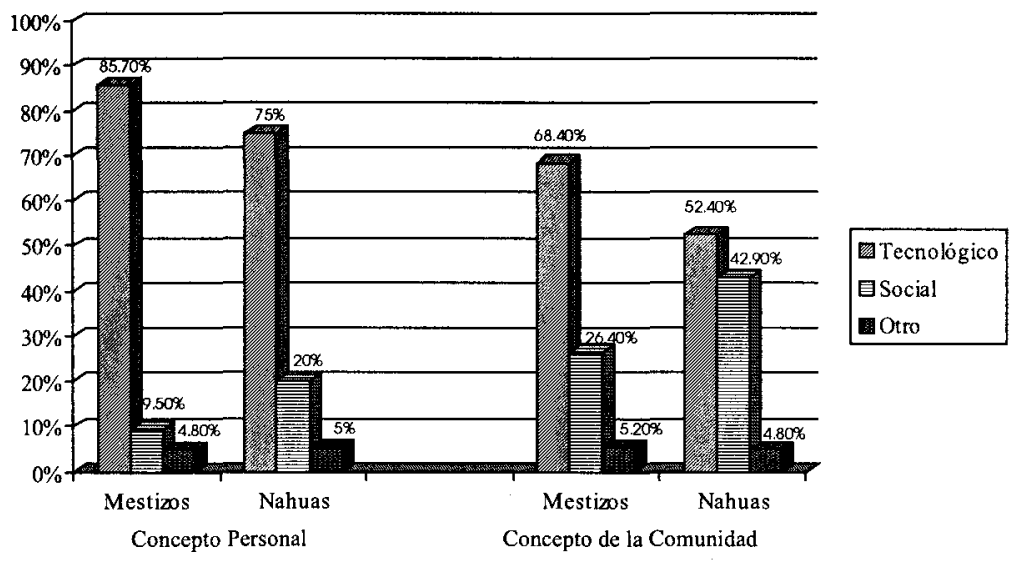

$N=21$

Figura 1. Porcentaje de conceptos proporcionados por los profesores mestizos y nahuas desde su perspectiva personal y desde la de la comunidad nahua. 
La Figura 1 muestra la distribución de los conceptos en cada una de las tres categorías, tanto para los profesores mestizos en lo que se refiere a su punto de vista personal y al punto de vista de la comunidad nahua, como para los profesores nahuas, también desde su propio punto de vista y desde el de la comunidad. Los profesores mestizos ofrecieron desde el punto de vista personal 18 conceptos que cayeron en la categoría de inteligencia tecnológica (85.7\%), 2 en la categoría de inteligencia social $(9.5 \%)$ y uno en la categoría otro $(4.8 \%)$. Por su parte, los profesores nahuas también desde el punto de vista personal generaron 15 conceptos $(75 \%)$ clasificados como tecnológicos, 4 sociales $(20 \%)$ y uno en la categoría otro $(5 \%)$. Cuando los profesores definieron la noción de niño inteligente desde la perspectiva de la comunidad nahua, los profesores mestizos manifestaron 13 conceptos tecnológicos de un total de $19(68.4 \%), 5$ sociales $(26.4 \%)$ y 1 en la categoría otro (5.2\%). En el caso de los profesores nahuas, se observó 11 conceptos tecnológicos $(52.4 \%), 9$ conceptos sociales $(42.9 \%)$ y uno en la categoría otro $(4.8 \%)$.

\section{ESTUDIO 2}

Tlane'neulistli: la inteligencia desde el punto de vista de las madres nahuas

Para conocer las nociones de personas inmersas en la comunidad (madres de familia) y ninguna escolaridad, se llevó a cabo un segundo estudio.

\section{Metodología}

\section{Participantes}

Participaron en el segundo estudio la totalidad de las 25 mujeres nahuas de la comunidad de Xilocuautla que cumplían con 
Concepto indígena de inteligencia en etnias de México

el siguiente criterio: madres de familia, con por lo menos dos hijos entre 6 y 12 años de edad. Todas habían nacido en la comunidad de Xilocuautla, carecían de experiencia escolar, hablaban náhuatl y, en menor medida, el español. Algunas de sus actividades principales consistían en cocinar, limpiar su casa, atender a los hijos y esposo, y colaborar para la subsistencia familiar realizando algunas tareas campesinas y vendiendo maíz, chile, tomate, col, leña o flores, en los días de mercado.

\section{Instrumentos}

Como primer paso en este estudio, se preguntó a 10 profesores rurales sobre el término empleado en náhuatl para referirse a la inteligencia, el mismo que resultó ser Tlane'neuilistli o Tlaniliuiski. Además, se preguntó a 10 mujeres nahuas, distintas a las participantes en el estudio, qué actividades suelen realizar los niños desde los 6 años de edad. La mayoría de los niños de la comunidad alimenta, cuida y asea a sus hermanos, limpia su casa, acarrea agua, busca y lleva leña, prepara alimentos, cuida animales, hace labores de siembra en el campo (como preparar la tierra, cultivar y cosechar), va a la escuela y hace sus tareas académicas.

Con dicha información se elaboró una tarea de selección de niño inteligente o tlane'neuilistli. La tarea se dividía en dos partes: una entrevista en náhuatl en la que las madres respondían a dos preguntas: a) ¿Conoce usted a niños inteligentes de su pueblo o de su familia o de familias que viven en Xilocuautla?, b) ¿Cómo son los niños inteligentes de su comunidad o de su familia o de familias de Xilocuautla? La segunda parte estaba compuesta por cuatro ilustraciones que representaban diferentes actividades realizadas por niños y niñas: a) un grupo de campesinos desempeñando su jornada, b) dos niños brincando la cuerda, c) un niño leyendo y otro escribiendo, d) un niño cargando leña, y e) una mujer y un niño recolectando chiles. 
Joaquín Figueroa Cuevas y Laura Hernández Guzmán

\section{Procedimiento}

Una vez contestadas ambas preguntas mediante entrevista individual, las madres identificaban, también individualmente, lo que estaban haciendo los niños en la ilustración y recibían la siguiente instrucción:

Todos los niños (el entrevistador señalaba la ilustración) tienen vacaciones, es decir, ya no van a la escuela. Estos niños juegan todo el tiempo (el entrevistador señalaba a los niños que aparecen brincando en el dibujo), estos niños se la pasan leyendo y escribiendo lo que les enseñaron en la escuela (el entrevistador señalaba a los niños que se hallaban leyendo y escribiendo), estos otros dos niños (el entrevistador señalaba a los niños que aparecían trabajando) pasan el tiempo ayudando a sus papás. Si todos estos niños fueran sus hijos (el entrevistador señalaba todos los niños que aparecían en la ilustración), ¿cuál sería para usted o su familia o su comunidad el niño más inteligente de todos ellos? ¿Por qué? Se anotaban todos los señalamientos y respuestas que dieran las madres.

\section{Resultados}

Cuatro de las participantes no respondieron a las preguntas. Las respuestas obtenidas de las 21 restantes fueron sí a la pregunta si conocían a un niño inteligente. Las respuestas a cómo era un niño Tlane'neuilistli o Tlaniliuiski en Xilocuautla cayeron en dos categorías: obediencia y responsabilidad (Figura 2). 
Concepto indígena de inteligencia en etnias de México

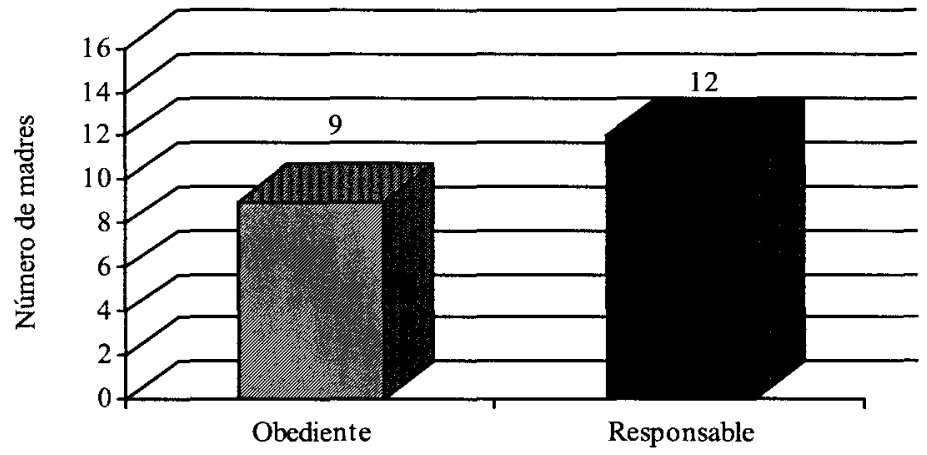

Conceptos

$$
N=21
$$

Figura 2. Porcentaje de madres nahuas $(N=21)$ que ofrecieron conceptos sociales o tecnológicos a la pregunta "¿Cómo son los niños inteligentes de su comunidad?".

La Figura 2 muestra que nueve de las 21 mujeres definieron a los niños y niñas inteligentes en términos de obediencia; por ejemplo:

- Son aquellos niños que se les dice que hagan un mandado y lo hacen sin protestar.

- $\quad$ Traen la leña, lavan trastos, ayudan en la siembra.

- Son los niños que no protestan cuando se les da un trabajo.

- Son aquellos que obedecen en los trabajos que les ponen a hacer los mayores. Los niños que no son inteligentes son los que no obedecen a sus padres, no los respetan.

Por otra parte, 12 lo hicieron en términos de responsabilidad. Algunos ejemplos son:

- Son los niños que cosechan mazorcas, buscan pastura para los burros, sin que se les diga, porque ellos ya saben lo que tienen que hacer. 
- Son los niños que se dan cuenta de lo que hace falta en la casa y se ponen a trabajar en eso.

- Son aquellos que ayudan a sus padres a trabajar sin explicarles tanto.

En la tarea de elección de una ilustración que identificaba lo que hacía un niño o niña inteligente, sí participaron las 25 madres. En la Figura 3 se aprecia que ninguna eligió a los niños jugando, 20 de ellas eligieron al niño que cargaba la leña o al niño que sembraba, justificaron su elección en términos de responsabilidad y obediencia.



Actividades de un niño int eligente

$$
\mathrm{N}=
$$

Figura 3. Porcentaje de madres nahuas $(N=25)$ que señalaron las actividades que realiza un niño o niña inteligente.

Cinco madres eligieron a los que estaban estudiando. Éstas justificaron su respuesta como sigue:

- Porque no dejan de aprender a leer y a escribir, pero también hay que enseñarles a trabajar en el campo no sólo en el estudio. Pero para mí los más listos son lo que estudian. 
Concepto indígena de inteligencia en etnias de México

- Los niños que están estudiando son los más inteligentes, porque lo que les enseñan en la escuela no se les olvida y lo repasan en la casa.

- Porque piensan y repasan lo que les enseñan en la escuela, además porque estudian para vivir mejor y no maltratarse como nosotros.

La noción de inteligencia de las madres nahua parece reflejar características predominantemente sociales. En primer lugar, porque todas mencionaron la responsabilidad y la obediencia como atributos fundamentales de los niños inteligentes o Tlane'neuilistli. Por otro lado, porque la mayoría seleccionó como ejemplo de niños inteligentes a los que trabajaban en labores campesinas, indicando, además, que eran responsables y obedientes.

\section{ESTUDIO 3}

Xanatli ix tanilat nak tachixitwit

(florecer es el modo de vida en comunidad): nociones de inteligencia de maestros bilingües

El último estudio pretendió investigar los conceptos de profesores pertenecientes a otras etnias mexicanas con respecto a su significado tecnológico o social.

\section{Metodología}

\section{Participantes}

Participaron la totalidad de 15 profesores bilingües representantes de regiones etnolinguísticas de nueve estados de la República Mexicana que asistían a un curso en la Ciudad de 
México. De Oaxaca: a'yuuk (mixe) media, chinanteca de Ojitlán, chinanteca de Usila y ngati'á (mazateca) y nanj ni’'in (triqui); de Chiapas: ch'ol y tojol'lab'al; de Tabasco: yokot'an (chontal); de Sonora: yoreme (mayo) y yoreme (yaqui); de Campeche, Yucatán y Quintana Roo: maya; de Guerrero: tu un savi (mixteca); de Puebla: nahua y totonaca; de San Luis Potosí: tének (huasteca). Todos ellos son nacidos y habitantes de comunidades indígenas con estudios de normal, dos de ellos con estudios de etnolingüística y cuatro egresados de la Universidad Pedagógica Nacional. La mayor parte de ellos son autores de los libros de texto de lenguas indígenas y responsables del desarrollo y evaluación de la educación indígena bilingüe en un programa denominado Escuelas de Asesoría.

\section{Procedimiento}

Se pidió a los maestros que respondieran por escrito, en forma individual, a dos preguntas:

1. ¿Cuáles son los componentes o partes que componen la inteligencia?

2. ¿Cómo es y qué hace en su comunidad un niño y una niña inteligentes?

Cada profesor podía contestar cada pregunta ofreciendo más de un concepto.

\section{Confiabilidad entre jueces}

Cuatro jueces independientes clasificaron la lista de conceptos ofrecidos por los profesores en tres categorías: inteligencia tecnológica, social y otro, al igual que en el estudio 1. Se calculó la confiabilidad por medio de la división de la suma de 
acuerdos entre la suma de acuerdos más desacuerdos, y se obtuvo, para los componentes que de acuerdo con su punto de vista personal eran parte de la inteligencia, una confiabilidad de .95 , y para los conceptos de inteligencia desde la perspectiva de la comunidad, una confiabilidad de .86 .

\section{Resultados}

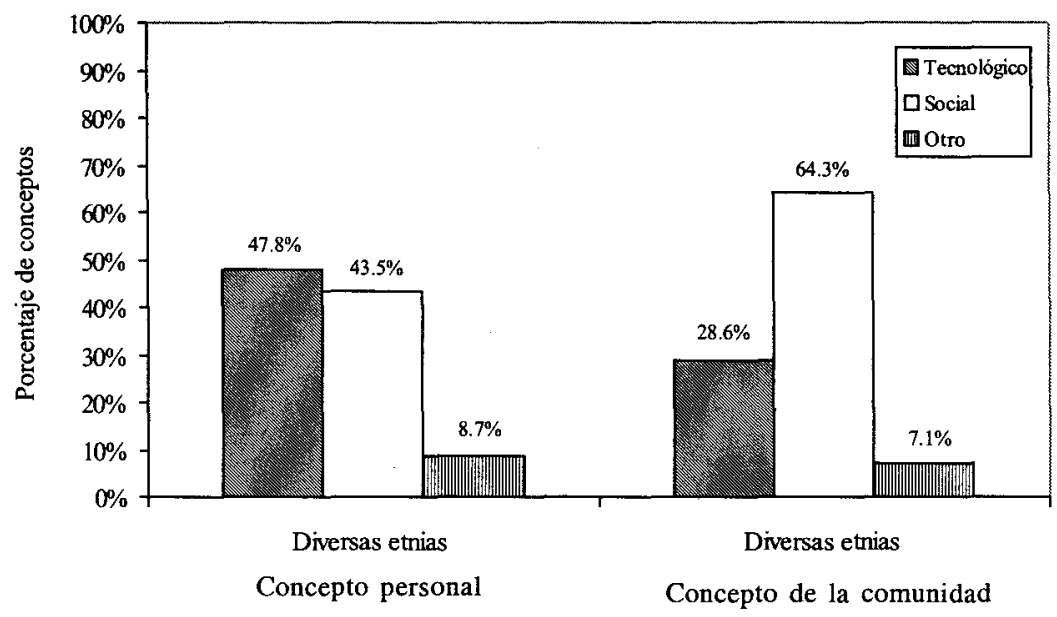

Figura 4. Porcentaje de conceptos proporcionados por los profesores de diversas etnias mexicanas desde su perspectiva personal y desde la de sus respectivas comunidades.

Se obtuvo un total de 23 conceptos de acuerdo con el concepto personal de los profesores, y 28 de acuerdo con el concepto de la comunidad. Se tomó en cuenta la totalidad de conceptos vertidos por los profesores, aunque se repitieran de un profesor a otro. La Figura 4 muestra los porcentajes de respuesta de los profesores a ambas preguntas. Respecto a la primera pregunta sobre los componentes que, de acuerdo con el juicio 
personal de los profesores, son parte de la inteligencia, 11 de un total de $23(47.8 \%)$ correspondieron a la categoría tecnológica, 10 $(43.5 \%)$ a la social y $2(8.7 \%)$ a otro. Ejemplos de estos componentes en la categoría tecnológica fueron: es listo, conoce, es reflexivo; en la categoría social: es respetuoso, ayuda a los demás, es cumplido; y en la categoría otros: es bueno, gana, es alegre.

Dieciocho de un total de $28(64.3 \%)$ de las respuestas a la pregunta desde la perspectiva de la comunidad fueron sociales, 8 (28.6\%) cayeron en la categoría tecnológica y $2(7.1 \%)$ en otro. Ejemplos de las respuestas a la pregunta sobre la perspectiva de la comunidad de un niño o niña inteligente, para la categoría social fueron: obedece a sus padres, sabe tratar a los demás, hace las cosas como se las piden sus papás sin esperar instrucciones. Ejemplos de respuestas en la categoría tecnológica fueron: comprende las cosas desde el primer momento, hace las cosas sin torpeza, y de la categoría otros: no es tímido.

\section{Discusión}

Los estudios 1 y 2 exploraron en la etnia nahua la noción de inteligencia, misma que parece variar de acuerdo con la escolaridad e influencia cultural externa. En el primer estudio, los profesores mestizos y los nahuas externaron, en un porcentaje mayor, un concepto tecnológico al definir la inteligencia desde su punto de vista personal. Cuando describieron la inteligencia desde la perspectiva de la comunidad nahua los conceptos tecnológicos disminuyeron y aumentaron los sociales ligeramente para los profesores mestizos. En el caso de los profesores nahuas, se observó también una reducción pequeña de los conceptos tecnológicos y un aumento de los conceptos sociales.

En el segundo estudio, las madres sin escuela expresaron predominantemente un concepto social. A diferencia de los 
profesores, las nociones de personas de la comunidad sin escolaridad (madres de familia) se inclinaron hacia una inteligencia social. Este resultado es congruente con los de investigaciones precedentes realizadas con grupos indígenas de otros continentes (Wober, 1974; Yang \& Sternberg, 1997), los que sugieren la importancia de los componentes sociales para la representación de la inteligencia desde la perspectiva indígena. La influencia del grado de contacto cultural de los profesores participantes en sus definiciones de inteligencia parece coincidir con los hallazgos documentados por Dasen et al. (1985) y Wober (1974). Al igual que Grigorenko et al. (2001), en el presente estudio los profesores, en comparación con los miembros de la comunidad, expresaron más atributos relacionados con las habilidades escolares. Las personas de la comunidad menos expuestas a la educación formal dieron mayor valor al aspecto social de la inteligencia. Una posible explicación sería que el contacto cultural con la sociedad occidental de los profesores tanto nahuas como mestizos del primer estudio influyera sobre su concepto esencialmente tecnológico de la inteligencia de los niños. Esta explicación emplea el concepto de contacto cultural y evita el concepto de aculturación que puede referir un desplazamiento absoluto e inmediato de la cultura autóctona por otra o la suma mecánica de culturas.

Por otra parte, al investigarse los conceptos de profesores pertenecientes a diversas etnias mexicanas, éstos parecen concordar más con la noción de los profesores nahuas y menos con la de los mestizos del primer estudio. No obstante, al responder lo que, desde la perspectiva de sus respectivas comunidades, era un niño o una niña inteligente, fueron más allá al dar respuestas predominantemente sociales, lo que coincide con las respuestas de las madres nahuas, quienes señalaron como más inteligentes a los niños que trabajaban en tareas domésticas. El tercer estudio repitió parcialmente en profesores de otras etnias lo hallado en la etnia nahua. Es precisamente el resultado del tercer 
estudio sobre la perspectiva de la comunidad, el que sugiere que diferentes etnias parecen compartir una noción de inteligencia social diferente a la occidentalizada, tal como lo muestra la literatura de investigación sobre una diversidad de etnias de regiones tan distantes entre sí como África, Asia y Latinoamérica.

En la interpretación de estos resultados es importante tomar en cuenta la situación en la que se da el lenguaje y las identidades étnicas. En el caso del lenguaje, la pragmática y con anterioridad a ésta la dialógica dan clara cuenta de la flexibilidad linguística para orientar el discurso de acuerdo con la situación social en la que se encuentre el hablante. Debido a lo anterior, puede explicarse la tendencia de las respuestas de los profesores a inclinarse más al lado social cuando la situación los refería a la inteligencia de acuerdo con la comunidad. Sus definiciones pasaron de los términos tecnológicos a los sociales de acuerdo con el contexto. Es posible también que los profesores indígenas tiendan a sobrestimar los atributos cognitivos y racionales. Esto podría conducir a situaciones de conflicto con respecto a la misión de la educación que se debate entre las contradicciones que existen entre sus objetivos económicos (la preparación del individuo para lograr éxito económico) y sus objetivos culturales (la preparación del individuo como perteneciente y continuador de una cultura) (Serpell, 1993).

Estos resultados sugieren que los nahuas y otros pueblos indígenas mexicanos conciben la inteligencia en forma social. El concepto social es más claro, al observar los resultados obtenidos de las madres nahuas sobre cómo son los niños nahuas considerados como Tlane'neulistli o Tlaniliuiski. En este caso, los niños inteligentes son los que ejecutan actividades fundamentales y útiles para la subsistencia familiar, en forma obediente y responsable. Es probable que consideren la responsabilidad y la obediencia como atributos inteligentes, porque expresan la capacidad de los niños para tomar conciencia de o pensar en las necesidades de subsistencia de su familia, saber lo que tienen que 
hacer al respecto sin que alguien se los diga y hacerlo ni protestar si alguien se los pide.

La obediencia y la responsabilidad como atributos intelectuales, también se han encontrado en otras partes del mundo (Berry, 1984; Dasen, 1984; Dasen et al., 1985). Al respecto Dasen señala que los resultados por él obtenidos en África sugieren que las aptitudes intelectuales individuales se valoran en la medida en que sirven a un objetivo social. El niño debe tener conocimientos, buena memoria, habilidad manual y debe saber hacer, pero solamente para el bien de la familia y la comunidad.

En esta misma línea de trabajo, Super (1983) refiere que para los adultos kipsigis (Kenia) la palabra gnom se utiliza para designar la inteligencia infantil y contiene un fuerte componente de responsabilidad. El ejemplo que este autor cita: "Una niña que es gnom barre la casa después de haber comido, porque ella sabe que así debe hacerse" (Super, 1983, p.317), recuerda las expresiones de las mujeres nahuas.

Los hallazgos referidos en la literatura de investigación en este campo, con los que coinciden los del presente estudio, tienen importantes consecuencias para el desarrollo de programas psicosociales y educativos en regiones etnolingüísticas. No resulta sorprendente que el desconocimiento de las diferencias de las prácticas culturales étnicas conduzca a educadores y psicólogos a interpretar la diversidad cultural como déficit intelectual de los niños, o a rechazar en la agenda educativa las competencias y conocimientos culturalmente significativos para los pueblos indígenas.

Desde la perspectiva de la psicología transcultural, el reto parece consistir en encontrar formas de evaluación más pertinentes, tomando en cuenta que los componentes sociales de la inteligencia apreciada por las culturas indígenas deben incorporarse al desarrollo de las pruebas de inteligencia. Por igual, es un reto para la construcción de planes curriculares 
dirigidos a población indígena incorporar competencias culturalmente valoradas que no poseen el mismo estatus o incluso sufren el menosprecio o el rechazo de las sociedades occidentales.

Se insiste en la necesidad de contar con instrumentos para la valoración de la inteligencia en los que se tome en cuenta el papel que se asigna a los atributos valorados localmente como componentes intelectuales y a subrayar la importancia que poseen los atributos sociales como la cooperación, responsabilidad y servicios a los otros como componentes básicos de las competencias, conocimientos y modalidad de interacción educativa que deben estar presentes en la atención escolar de los niños indígenas. Igualmente, es relevante para las políticas educativas de países que, como Estados Unidos, enfrentan un flujo de inmigración de diversos grupos indígenas provenientes de México y otros países latinoamericanos.

El conocimiento de los conceptos de inteligencia y sus puntos de coincidencia podrían ser el punto de partida para la construcción de pruebas de inteligencia más válidas y, por lo tanto, más equitativas y justas.

Los tres estudios presentan limitaciones que obligan a considerar con cautela las conclusiones discutidas arriba. A pesar de que se trabajó con la totalidad de la población en la comunidad y de los profesores disponibles en el momento de la recolección de los datos, el número de participantes resultó muy reducido, lo que afecta la generalidad y el alcance de la interpretación de estos resultados. Debido al acceso limitado a estas comunidades por parte de extraños, en el caso del presente estudio sólo se logró trabajar con una comunidad. Los estudios futuros deberán emplear muestras representativas compuestas por un mayor número de participantes, probablemente tanto de la etnia nahua como de otras para realizar comparaciones entre éstas. Esto permitirá un tratamiento más riguroso de los datos y análisis que permitan un mayor nivel de inferencia. Asimismo, sería interesante comparar sistemáticamente los conceptos de inteligencia de sociedades 
Concepto indígena de inteligencia en etnias de México

indígenas con los de grupos de mestizos sin contacto alguno con éstas, así como de diversos grupos etáreos.

\section{Referencias}

Berry, J. W. (1984). Towards a universal psychology of cognitive competence. International Journal of Psychology, 19, 335361 .

Cole, M. \& Scribner, S. (1977). Cultura y pensamiento: relación de los procesos cognoscitivos con la cultura. México: Limusa.

Chamoux, M. (1987). Nahuas de Huauchinango. Transformaciones sociales en una comunidad campesina. México: INI-CEMCA.

Church, T. A. \& Katigbak, M. S. (1988). Imposed etic and emic measures of intelligence as predictors of early school performance of rural Philippine children. Journal of CrossCultural Psychology, 19(2), 164-177.

Dasen, P. R. (1984). The cross-cultural study of intelligence: Piaget and the Baoulé. International Journal of Psychology, 19, 407-434.

Dasen, P. R., Barthélémy, D., Kan, E., Kouamé, K., Daouda, K., Adjéi, K.K. et al. (1985). N'Glouele, l'intelligence chez les Baoulé. Archives de Psychologie, 53, 293-324.

Grigorenko, E. L., Geissler, P. W., Prince, R., Okatcha, F., Kenny, D. A., Bundy, D. A. et al. (2001). The organization of Luo conceptions of intelligence: A study of implicit theories in a Kenyan village. International Journal of Behavioral Development, 25(4), 367-378.

Kathuria, R. \& Serpell, R. (1998). Standardization of the Panga Munthu test: A nonverbal cognitive test developed in Zambia. Journal of Negro Education, 67(3), 228-241.

Klein, R. H., Freeman, H. \& Millet, R. (1973). Psychological tests 
performance and indigenous conceptions of intelligence. Journal of Social Psychology, 84, 219-222.

Price-Williams, D., Gordon, W. \& Ramirez, M. (1969). Skill and conservation. A study of pottery-making children. Developmental Psychology, 1, 769.

Serpell, R. (1977). Estimates of intelligence in a rural community of Eastern Zambia. En F. M. Okatcha (Ed.), Modern psychology and cultural adaptation (pp. 179-216). Nairobi:

Swahili Language Consultants and Publishers.

Serpell, R. (1984). Commentary: The impact of psychology on the third world development. International Journal of Psychology, 19, 179-192.

Serpell, R. (1993). The significance of schooling: Life-journeys in an African society. Lusaka: University of Zambia.

Sternberg, R. J. (1985). Human intelligence: The model is the message. Science, 230(4730), 1111-1118.

Sternberg, R. J., Conway, B. E., Ketron, J. L. \& Bernstein, M. (1981). People's conceptions of intelligence. Journal of Personality and Social Psychology, 41(1), 37-55.

Sternberg, R. J. \& Grigorenko, E. L. (1997). Infamous artifacts in the study of intelligence. Why there is so much support for so many hypotheses. Cahiers de Psychologie Cognitive, 16(6), 769-778.

Sternberg, R. J., Grigorenko, E. L., Ngurosho, D., Tantufuye, E., Mbise, A., Nokes, C. et al. (2002). Assessing intellectual potential in rural Tanzanian school children. Intelligence, 30(2), 141-162.

Super, C. M. (1983). Cultural variation in the meaning and uses of children's “intelligence”. En J. Deregowski, S. Dziurawiec \& R. Annis (Eds.), Explications in cross-cultural psychology. Amsterdam: Swets and Zeitlinger.

Wober, M. (1974). Towards an understanding of the Kiganda concept of intelligence. En J. W. Berry \& P. R. Dasen (Eds.), 
Concepto indígena de inteligencia en etnias de México

Culture and cognition: Readings in cross-cultural psychology. Londres: Methuen and Co.

Yang, S.Y. \& Sternberg, R. J. (1997). Conceptions of intelligence in ancient Chinese philosophy. Theoretical \& Philosophical Psychology, 17(2), 101-119. 